\title{
High-Response i-InAs/n-GaAs Quantum-Dot Infrared Photodetector with No Current Blocking Barrier
}

\author{
Sang Jun Lee, Sam Kyu NoH*, Jung Woo ChOE ${ }^{1}$, Uk Hyun $\mathrm{LeE}^{2}$, Sung Chul $\mathrm{HONG}^{2}$ and Jung Il $\mathrm{LeE}^{3}$ \\ Quantum Dot Technology Laboratory, Korea Research Institute of Standards and Science, Daedeok Science Town, Daejeon 305-600, Korea \\ ${ }^{1}$ Department of Electrical Engineering and Computer Science, Kyunghee University, Suwon 449-701, Korea \\ ${ }^{2}$ Department of Electrical Engineering and Computer Science, Korea Advanced Institute of Science and Technology, Daejeon 305-701, Korea \\ ${ }^{3}$ Nano-Device Research Center, Korea Institute of Science and Technology, Seoul 136-791, Korea
}

(Received October 10, 2003; accepted November 25, 2003; published March 10, 2004)

\begin{abstract}
We report a high-response normal-incidence infrared photodetector based on the bound-to-continuum transition fabricated on the self-assembled i-InAs/n-GaAs quantum-dot (QD) heterostructure doped in GaAs barrier with no current blocking barrier. The photoresponse characteristics have been confirmed by distinct methods using a SiC globar source and a blackbody radiation source. From the blackbody system, the responsivity of $1750 \mathrm{~mA} / \mathrm{W}$ at a peak wavelength of $\sim 4.7 \mu \mathrm{m}(21 \mathrm{~K})$ has been achieved, which is much higher than that reported on the n-InAs/i-GaAs QD-based infrared photodetectors doped in QDs with additional barrier. [DOI: 10.1143/JJAP.43.1218]
\end{abstract}

KEYWORDS: indium arsenide, quantum dot, infrared photodetector, responsivity, detectivity

\section{Introduction}

Quantum-dot infrared photodetector (QDIP) and its related heterostructures have been intensively studied in order to realize normal-incidence room-temperature operation overcoming the limitation of quantum well (QW)based devices. ${ }^{1-17)}$ In spite of the theoretical prediction on QDIPs possessing distinguished infrared (IR) detector performance, ${ }^{18-20)}$ the responsivity $(R)$ and the specific detectivity $\left(D^{*}\right)$ of QDIPs stay at $\sim 10^{2} \mathrm{~mA} / \mathrm{W}$ and $\sim 10^{9} \mathrm{~cm} \cdot \mathrm{Hz}^{1 / 2} / \mathrm{W}$ around $100 \mathrm{~K}$ in the mid-IR range, ${ }^{1-11)}$ respectively, which are a little inferior to QWIPs. Of current necessity, therefore, is a QDIP device with enhanced performance operating at higher temperatures in longer spectral range near the atmospheric window $(8-12 \mu \mathrm{m})$. Stiff et al. demonstrated characteristics of individual QDIP device operation as high as $150 \mathrm{~K}$ in the mid-IR range peaking at $\lambda_{\mathrm{p}} \sim 3.7 \mu \mathrm{m}$ together with a raster-scanned image realized by $(13 \times 13)$ array. ${ }^{1)}$ Recently, Tang et al. reported a QDIP device of photoconductive and photovoltaic mixed mode working up to $250 \mathrm{~K}$ in the range of $2-5 \mu \mathrm{m},{ }^{2,3)}$ and Krishna et al. presented a two-color detector $\left(\lambda_{\mathrm{p}} \sim 4.2 / 7.6 \mu \mathrm{m}\right) \mathrm{using}$ InAs/InGaAs dot-in-well (DWELL) structure. ${ }^{4)}$ They have adopted modified QDIP structures with a single/double AlGaAs blocking layer or a DWELL structure in which the dark current reduction or better carrier confinement can be expected by the additional potential barrier, as opposed to a drawback of photocurrent sacrifice.

In this letter, we report an achievement of high values of $R \sim 2000 \mathrm{~mA} / \mathrm{W}$ and $D^{*} \geq 10^{9} \mathrm{~cm} \cdot \mathrm{Hz}^{1 / 2} / \mathrm{W}(21 \mathrm{~K})$ at a peak wavelength of $\lambda_{\mathrm{p}} \sim 5.0 \mu \mathrm{m}$ obtained from an i-InAs $/ \mathrm{n}$ GaAs QDIP structure doped in GaAs barrier with no current blocking barrier. It shows a quite broad spectral photoresponse over the far-IR wavelength of $3-10 \mu \mathrm{m}$ operating at temperatures up to around $200 \mathrm{~K}$. In order to confirm the device characteristics, the photoresponse measurements have been done by a couple of distinct methods using a $\mathrm{SiC}$ globar source dispersed by the far-IR spectrometer and a well-calibrated blackbody radiation source combined with a set of bandpass filters. We present the power dependence of

${ }^{*}$ Corresponding author. E-mail address: sknoh@kriss.re.kr micro-photoluminescence ( $\mu$-PL) spectra as evidence indicating the bound-to-continuum transition in the QDIP operation.

\section{Experimental Procedure}

The molecular beam epitaxy (MBE) technique was introduced for the growth of QDIP structure, and the selfassembled QD ensemble was realized via the StranskiKrastanow (S-K) growth mode. The $\mathrm{n}-\mathrm{i}-\mathrm{n}$ QDIP device prepared for this study has an active layer stacked by five periods of i-InAs-QD/n-GaAs:Si, and the Si doping in GaAs barrier was adopted for effective IR absorption proven by previous studies. ${ }^{12)}$ The growth was conducted at temperatures of $580^{\circ} \mathrm{C}$ from $\mathrm{GaAs}$ buffer to bottom contact layer and of $460^{\circ} \mathrm{C}$ from the active region to top contact layer. Each layer of InAs QDs with an equivalent thickness of 2.5 monolayers (MLs) was formed on undoped GaAs spacer, and a 3-nm-thick doped layer $\left(1 \times 10^{17} \mathrm{~cm}^{-3}\right)$ was positioned at $6 \mathrm{~nm}$ above the InAs-QD layer in the $40-\mathrm{nm}$ GaAs spacer for each stack. Taking care of the QD density, the layer thickness and the doping level were designed in order that each dot was occupied by approximately one electron. The thick GaAs overlayer was introduced in the active medium to induce spatially random distribution with broader spectral response. The epitaxial growth and basic properties on the related QD heterostructures are described in detail in previous reports. ${ }^{12-15)}$

The QD formation was confirmed by a cross-sectional image of the transmission electron microscope (TEM) as shown in the inset of Fig. 1, and the dot density of $\sim 5 \times$ $10^{10} \mathrm{~cm}^{-2}$ was identified from the atomic force microscopy (AFM) profile of an uncapped QD structure. ${ }^{12)}$ Circular-ring and square shaped electrodes were formed on top and bottom $\mathrm{n}^{+}$-GaAs layers, respectively, by using the standard photolithography and the wet-chemical etching procedures, and the diameter of the illumination opening for individual device was $450 \mu \mathrm{m}\left(A=1.6 \times 10^{5} \mu \mathrm{m}^{2}\right)$. A couple of distinct measurements were independently performed at the normal-incidence configuration. One is the continuousspectrum method using a broadband globar source dispersed by the far-IR spectrometer with a pair of gratings of 60 and 120 grooves/mm and calibrated by a $\mathrm{HgCdTe}$ detector. The 


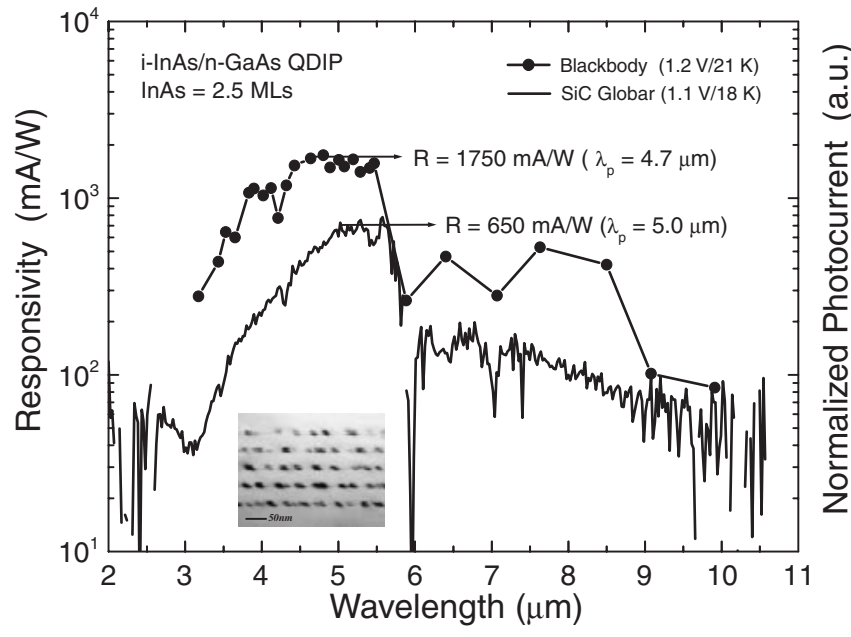

Fig. 1. The responsivity data $(1.2 \mathrm{~V} / 21 \mathrm{~K}$, solid circles) by blackbody source and the normalized photocurrent spectrum $(1.1 \mathrm{~V} / 18 \mathrm{~K}$, solid line) which directly corresponds to the responsivity by globar source. Both curves show similar IR detection behavior over the spectral range of $3-$ $10 \mu \mathrm{m}$ within the filter bandwidth of $\sim 0.2 \mu \mathrm{m}$.

other is the point-by-point measurement by a combination of a well-calibrated commercial blackbody radiation source (IR-564, Infrared System Development Corp.) and a set of bandpass filters (OCLI). The filter set used in this experiment consists of 43 filters with a narrow bandwidth of $\sim 0.2 \mu \mathrm{m}$ in the range of $3-14 \mu \mathrm{m}$. A typical visible-to-nearIR monochromator system with a $\mathrm{He}-\mathrm{Ne}(632.8 \mathrm{~nm})$ laser and a liquid-nitrogen cooled Ge photodetector was used in the $\mu$-PL measurements.

\section{Results and Discussion}

The responsivity data $(1.2 \mathrm{~V} / 21 \mathrm{~K}$, solid circles $)$ by the blackbody source are compared in Fig. 1 with a photocurrent spectrum $(1.1 \mathrm{~V} / 18 \mathrm{~K}$, solid line) by the globar source normalized to the spectral photon flux, which directly corresponds to the responsivity. Both curves show similar IR response behavior over the spectral range of $3-10 \mu \mathrm{m}$ peaking around $4.5-5.5 \mu \mathrm{m}$, and are consistent with each other within the filter bandwidth of $\sim 0.2 \mu \mathrm{m}$. The peak values of $R$ and equivalent $D^{*}$ are $1750 / 650 \mathrm{~mA} / \mathrm{W}$ and $1.2 \times 10^{9} / 3.2 \times 10^{8} \mathrm{~cm} \cdot \mathrm{Hz}^{1 / 2} / \mathrm{W}$ for the blackbody/globar source, respectively. (For reference, $R / D^{*}=2 \mathrm{~mA} / \mathrm{W} /$ $2.9 \times 10^{9} \mathrm{~cm} \cdot \mathrm{Hz}^{1 / 2} / \mathrm{W}\left(T=100 \mathrm{~K}, \lambda_{\mathrm{p}}=3.7 \mu \mathrm{m}\right), 155 \mathrm{~mA} /$ $\mathrm{W} / 2.3 \times 10^{9} \mathrm{~cm} \cdot \mathrm{Hz}^{1 / 2} / \mathrm{W} \quad\left(T=36 \mathrm{~K}, \quad \lambda_{\mathrm{p}}=2.8 \mu \mathrm{m}\right)$, and $730 \mathrm{~mA} / \mathrm{W} / 1.5 \times 10^{9} \mathrm{~cm} \cdot \mathrm{Hz}^{1 / 2} / \mathrm{W} \quad\left(T=60 \mathrm{~K}, \quad \lambda_{\mathrm{p}}=\right.$ $4.2 \mu \mathrm{m})$ for Stiff et al., ${ }^{1)}$ Tang et al., ${ }^{2,3)}$ and Krishna et al., ${ }^{4)}$ respectively.) The value of $R$ obtained in this study is much higher than the previously reported data as enumerated just above, and the $D^{*}$ value is fairly high taking consideration of no current blocking barrier. The high $R$-value indicates that the present $\mathrm{i}-\mathrm{InAs} / \mathrm{n}-\mathrm{GaAs}$ QDIP structure doped in GaAs barrier is very effective for IR absorption.

Figure 2 presents the dependences of $D^{*}$ on temperature (open circles) and bias voltage (open squares) for the globar system together with the corresponding data (solid circle/ square, $1.2 \mathrm{~V} / 21 \mathrm{~K}$ ) for the blackbody system. The two curves monotonically decrease with both increasing temperature (bottom scale) and decreasing bias voltage (top scale), and survive up to $190 \mathrm{~K}$ and down to $0.8 \mathrm{~V}$. The misfit

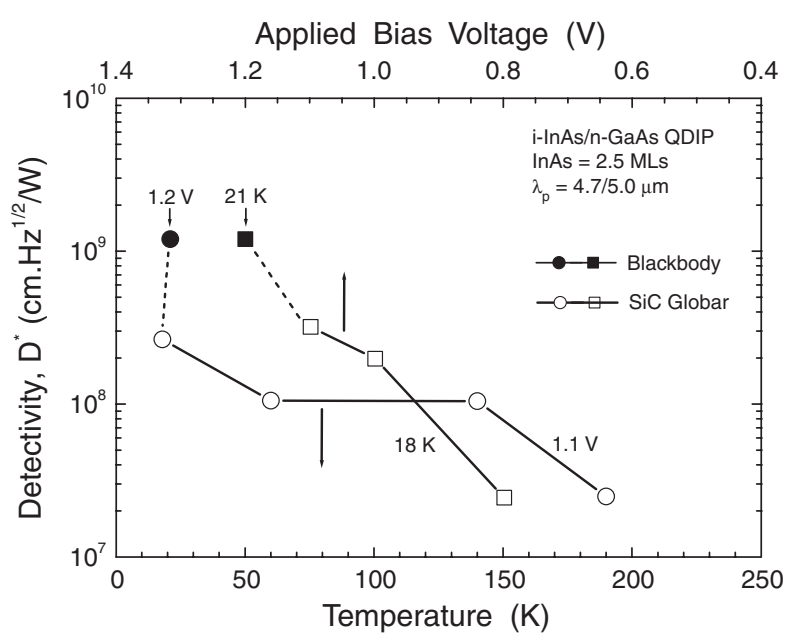

Fig. 2. Temperature (open circles) and bias voltage (open squares) dependences of $D^{*}$ by the globar system, and the corresponding value (solid circle/square) by the blackbody system. The two curves monotonically decrease with both increasing temperature (bottom scale) and decreasing bias voltage (top scale), and survive up to $190 \mathrm{~K}$ and down to $0.8 \mathrm{~V}$.

between two $D^{*}$-values for blackbody/globar sources is partially due to the difference in the noise frequency range. In determining the $D^{*}$ 's, we have used the current noise density measured at high frequency $\left(60 \mathrm{pA} / \mathrm{Hz}^{1 / 2}\right.$ at $\left.6 \mathrm{kHz}\right)$ and low frequency $\left(80 \mathrm{pA} / \mathrm{Hz}^{1 / 2}\right.$ at $\left.200 \mathrm{~Hz}\right)$ for blackbody and globar sources, respectively, because of the different experimental setup. Therefore, the $D^{*}$ value raised by $4 / 3$ can be expected if we apply the high-frequency noise density to $D^{*}$ for globar source. We think that the remaining misfit in $D^{*}$ 's may be possibly attributed to the spectrometer system including a $\mathrm{HgCdTe}$ detector used for the photon flux calibration of globar source. This indicates that the choice of the dynamic frequency range for signal recovery and the photon flux calibration in the far-IR spectrometer system using globar source have to be carefully done in the manipulation of $D^{*}$. Table $\mathrm{I}$ is a comparative summary of normal-incidence characteristics on the QDIP device measured by the blackbody radiation source and the $\mathrm{SiC}$ globar source.

Finally, in order to verify that the photoresponse of QDIP originates from the bound-to-continuum transition, we have investigated the $\mu$-PL spectra $(77 \mathrm{~K})$ for various measurement conditions and QDIP samples. Figure 3 exhibits two sets of $\mu$-PL spectra taken (a) at three different excitation-

Table I. The comparative summary of the normal-incidence device characteristics of an $\mathrm{i}-\mathrm{InAs} / \mathrm{n}-\mathrm{GaAs}$ QDIP structure measured by the blackbody radiation and the $\mathrm{SiC}$ globar sources.

\begin{tabular}{lcc}
\hline Infrared Source & Blackbody Radiation & SiC Globar \\
\hline Spectrometer System & Bandpass Filters & Gratings \\
Operation Voltage $(\mathrm{V})$ & $(\Delta \lambda \sim 0.2 \mu \mathrm{m})$ & $(60 / 120 \mathrm{gr} / \mathrm{mm})$ \\
Measurement Temperature $(\mathrm{K})$ & 1.2 & 1.1 \\
Peak Wavelength $(\mathrm{mm})$ & 21 & 18 \\
Peak Responsivity $(\mathrm{mA} / \mathrm{W})$ & 4.7 & 5.0 \\
Noise Current Density $\left(\mathrm{pA} / \mathrm{Hz}^{1 / 2}\right)$ & $60($ at $6 \mathrm{kHz})$ & $80($ at $200 \mathrm{~Hz})$ \\
Peak Detectivity $\left(\mathrm{cm} \cdot \mathrm{Hz}^{1 / 2} / \mathrm{W}\right)$ & $1.2 \times 10^{9}$ & $3.2 \times 10^{8}$ \\
\hline
\end{tabular}




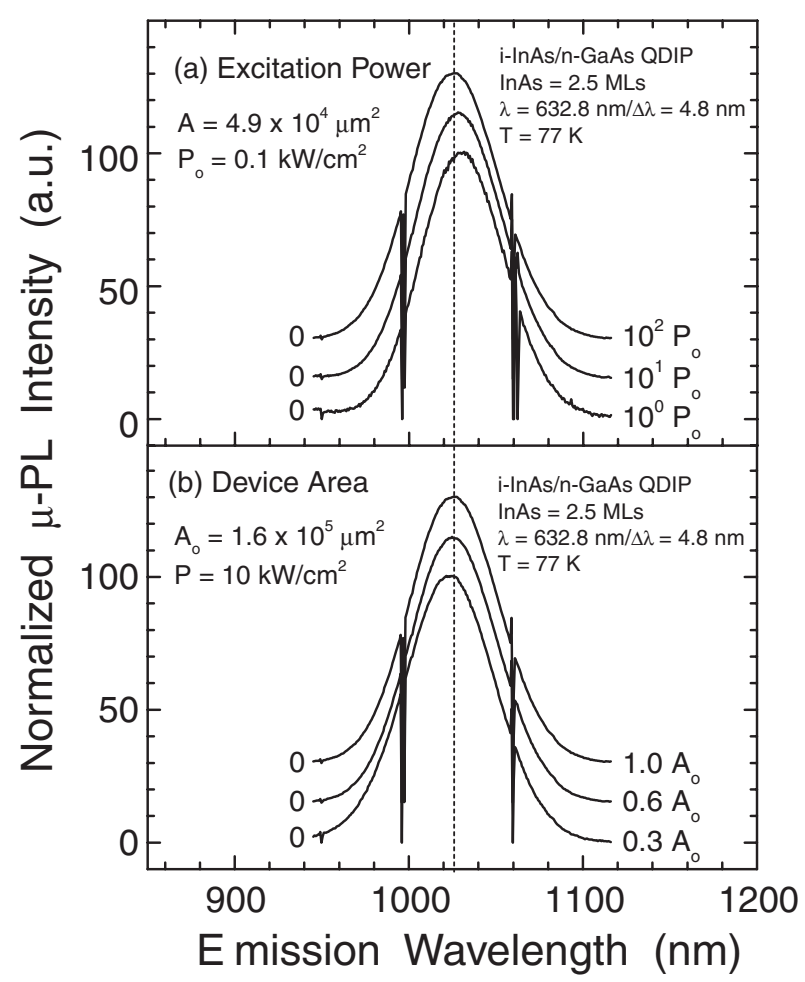

Fig. 3. Two sets of $\mu$-PL spectra $(77 \mathrm{~K}$ ) taken (a) at three different excitation-power densities and (b) from three kinds of devices with different area. All the spectra clearly show almost no changes in peak energies and spectral shapes.

power densities of $0.1-10 \mathrm{~kW} / \mathrm{cm}^{2}$ and (b) from three kinds of devices with different areas of $0.5-1.6 \times 10^{5} \mu \mathrm{m}^{2}$ simultaneously fabricated on an identical wafer. All the spectra clearly show almost no changes in peak energies and spectral shapes, which suggest that there exists no excited state in QDs. It indicates that the photoresponse data presented in this study may be possibly due to the boundto-continuum transition of QD ensemble.

\section{Summary and Conclusions}

In summary, we reported an achievement of highly effective normal-incidence mid-IR QDIP with $R=$ $1750 \mathrm{~mA} / \mathrm{W}$ and $D^{*}=1.2 \times 10^{9} \mathrm{~cm} \cdot \mathrm{Hz}^{1 / 2} / \mathrm{W}$ at $\lambda_{\mathrm{p}} \cong$ $4.7 \mu \mathrm{m}(21 \mathrm{~K})$ in the $\mathrm{i}-\mathrm{InAs} / \mathrm{n}-\mathrm{GaAs}$ QD heterostructure doped in GaAs barrier with no current blocking barrier. We confirmed that the mid-IR absorption originated from the bound-to-continuum transition of QD ensemble through the power dependence of $\mu$-PL spectra. The value of $R$ was much higher than that reported on n-InAs/i-GaAs QDIP structures with additional barrier. We suggested that the mid-IR response of $\mathrm{i}-\mathrm{InAs}-\mathrm{QD} / \mathrm{n}-\mathrm{GaAs}$ QDIP device doped in GaAs barrier was very efficient in comparison with n-InAs-QD/i-GaAs structures doped in QDs.

\section{Acknowledgments}

This work was supported in part by MOIC (Contract No. IMT2000-B4-1), and mainly carried out at the National Research Laboratory on Quantum Dot Technology at KRISS designated by MOST (Contract No. M1-0104-00-0127). One of the authors (SKN) acknowledges the partial support provided by KOSEF through the Quantum-Functional Semiconductor Research Center at Dongguk University in 2003.

1) A. D. Stiff-Roberts, S. Chakrabarti, S. Pradhan, B. Kochman and Bhattacharya: Appl. Phys. Lett. 80 (2002) 3265.

2) S.-F. Tang, S.-Y. Lin and S.-C. Lee: Appl. Phys. Lett. 78 (2001) 2428

3) S.-F. Tang, S.-Y. Lin and S.-C. Lee: IEEE Trans. Electron Devices 49 (2002) 1341

4) S. Krishna, S. Raghaven, G. von Winckel, P. Rotella, A. Stintz, C. P. Morath, D. Le and S. W. Kennerly: Appl. Phys. Lett. 82 (2003) 2574.

5) D. Pan, E. Towe and S. Kernnerly: Appl. Phys. Lett. 76 (2000) 3301.

6) S. Y. Wang, S. D. Lin, H. W. Wu and C. P. Lee: Appl. Phys. Lett. 78 (2001) 1023.

7) Z. Chen, E.-T. Kim and A. Madhukar: Appl. Phys. Lett. 80 (2002) 2490 .

8) Z. Ye, J. C. Campbell, Z. Chen, E.-T. Kim and A. Madhukar: J. Appl. Phys. 92 (2002) 4141.

9) S.-Y. Lin, Y.-J. Tsai and S.-C. Lee: Jpn. J. Appl. Phys. 40 (2001) L1290.

10) S.-W. Lee, K. Hirakawa and Y. Shimada: Appl. Phys. Lett. 75 (1999) 1428.

11) N. Horiguchi, T. Futatsugi, Y. Nakata, N. Yokoyama, T. Mankad and P. M. Petroff: Jpn. J. Appl. Phys. 38 (1999) 2559.

12) S. K. Kang, S. J. Lee, J. I. Lee, M. D. Kim, S. K. Noh, Y. H. Kang, U. H. Lee, S. C. Hong, H. S. Kim and C. G. Park: J. Korean Phys. Soc. 42 (2003) 418.

13) M. D. Kim, S. K. Noh, S. C. Hong and T. W. Kim: Appl. Phys. Lett. 82 (2003) 553.

14) M. D. Kim, S. K. Noh, S. C. Hong and T. W. Kim: Virtual J. Nanoscale Sci. Technol. 7 (2003).

15) J. S. Kim, P. W. Yu, J. Y. Leem, J. I. Lee, S. K. Noh, J. S. Kim, S. M. Kim, J. S. Son, U. H. Lee, J. S. Yim and D. Lee: Appl. Phys. Lett. 78 (2001) 3247.

16) G. Park, O. B. Shchenkin, S. Csutak, D. L. Huffaker and D. G. Deppe: Appl. Phys. Lett. 75 (1999) 3267.

17) S.-Y. Lin, Y.-J. Tsai and S.-C. Lee: Jpn. J. Appl. Phys. 40 (2001) L1290.

18) J. Phillips: J. Appl. Phys. 91 (2002) 4590.

19) V. Ryzhil: Semicond. Sci. Technol. 11 (1996) 759.

20) V. Ryzhil: Jpn. J. Appl. Phys. 40 (2001) L148. 